\title{
Smoking attributable medical expenditures, years of potential life lost, and the cost of premature death in Taiwan
}

\author{
M C Yang, C Y Fann, C P Wen, T Y Cheng
}

Tobacco Control 2005; 14(Suppl I):i62-i70. doi: 10.1136/tc.2004.007963

\begin{abstract}
See end of article for authors' affiliations

....................

Correspondence to: Ming-Chin Yang, Institute of Health Care

Organization

Administration, National

Taiwan University, Taipei,

Taiwan; mcy@ha.mc.ntu. edu.tw
\end{abstract}

\begin{abstract}
Objective: To estimate the smoking attributable medical expenditures and productivity loss of people aged 35 and over in Taiwan in 2001 from a societal viewpoint.

Methods: A prevalence based approach was used to estimate smoking attributable costs. Epidemiological parameters were obtained from two follow up studies and government statistics. Data on medical care utilisation and expenditure were extracted from the National Health Insurance claim data.

Results: Total smoking attributable medical expenditures (SAEs) amounted to US\$397.6 million, which accounted for $6.8 \%$ of the total medical expenditures for people aged 35 and over. Mean annual medical expenditures per smoker was US $\$ 70$ more than that of each non-smoker. Smoking attributable years of potential life lost (YPLL) totalled to 217761 years for males and 15462 years for females, and the corresponding productivity loss was US\$1371 million for males and US\$18.7 million for females.

Conclusion: Medical expenditures attributable to smoking accounted for $6.8 \%$ of the total medical expenditure of people aged 35 and over for the year 2001 in Taiwan. Corresponding YPLL and productivity loss also demand that actions be taken to fight cigarette smoking.
\end{abstract}

S moking is considered to be an important cause of premature mortality and disability. The World Health Organization estimated that annually smoking caused about four million deaths worldwide. ${ }^{1}$ The US Centers for Disease Control and Prevention also reported that annually smoking caused approximately 444000 premature deaths and cost $\$ 157$ billion in health related economic losses in the USA between 1995 and $1999 .^{2}$

Although numerous studies already reported the association between tobacco and disease and huge attributable expenditures, the association gained more attention due to recent tobacco settlements in the USA. To date, economic value of tobacco is still being debated between public health advocators and the tobacco industry. ${ }^{3}$

In Taiwan, health authorities have made controlling and preventing the adverse effects of smoking an important priority and enacted the Tobacco Hazards Control Act in 1997. During the policy formulation process, the authors conducted several studies to estimate smoking attributable expenditures (SAEs) in an attempt to provide evidence of the adverse effects of cigarette smoking. However, due to limited local data, certain parameters in our previous estimations were forced to use values reported in studies from other countries.

Fortunately, many of those data are now available after the implementation of the National Health Insurance (NHI) in 1995. Being a compulsory social insurance, more than $97 \%$ of the population were covered under this programme. The Bureau of NHI has released selected claims data for academic use since 2001. Therefore, types of services used and the medical expenditures can be determined from the datasets. In addition, epidemiological data were available by merging two cohort datasets-one was provided by Liaw et al (a community cohort) ${ }^{5}$ and the other by Wen et al (a civil servant and teacher cohort). ${ }^{67}$ A total of 86580 persons were included in the final dataset. The mortality risks of current smokers compared to non-smokers were calculated based on Cox's proportional hazards model adjusted by age. Detailed descriptions of these studies and the relative risks (RR) were reported elsewhere. ${ }^{7}$ Based on the information gathered from the above mentioned sources, we can estimate the SAEs more precisely.

The purpose of this study, therefore, was to estimate the SAEs in Taiwan in 2001 from a societal viewpoint. The results of this study could serve as a reference for health authorities when formulating tobacco control policies, and be used in health education programmes for the public.

\section{METHODS}

We used the prevalence based approach to estimate the burden of smoking on the society in 2001. Human capital approach was adopted as the theoretical basis to estimate cost of productivity caused by premature death. The method suggested by Rice et al ${ }^{8}$ was used to estimate excess costs due to smoking in a year based on epidemiological findings.

Regarding the definition of smoking status, we followed the definitions used by Wen $e t a^{7}$ that current smokers were those who were still smoking at the time of recruitment into the cohort study; non-smokers were those who never smoked; and ex-smokers were those who had quit smoking at least six months before the study.

In terms of parameters needed to estimate SAEs, we first estimated RRs of developing smoking related diseases between current smokers and non-smokers, followed by estimating the amount of medical care used and expenditures associated with each smoking related disease. The followings are detailed descriptions of how the parameters and costs were estimated in this study.

\section{Estimating smoking attributable fraction (SAF)}

We used smoking attributable fraction (also known as population attributable risk) to estimate the quantity of medical care attributable to smoking. This concept was first

\footnotetext{
Abbreviations: ICD-9, International classification of diseases, $9^{\text {th }}$ revision; $\mathrm{NHI}$, National Health Insurance; RR, relative risk; SAD, smoking attributable deaths; SAE, smoking attributable medical expenditure; SAMMEC II, smoking attributable mortality, morbidity, and economic costs software, release II; SAPDC, smoking attributable cost of premature deaths; SAYPLL, smoking attributable years of potential life lost; SFA, smoking attributable fraction; YPLL, years of potential life lost
} 
defined by Levin as the "maximum proportion of lung cancer attributable to cigarette smoking". ${ }^{9}$ Subsequently, the concept was renamed as smoking attributable fraction (SAF) and used by Rice et $a l^{8}$ and Shultz et al. ${ }^{10}$ Below is the formula used to calculate SAF in this study:

$$
\begin{aligned}
\mathrm{SAF}_{i y s}= & {\left[\mathrm{P}^{0}{ }_{i y s}+\mathrm{P}^{1}{ }_{i y s}^{*} \mathrm{RR}^{1}{ }_{i y s}+\mathrm{P}^{2}{ }_{i y s}{ }^{*} \mathrm{RR}^{2}{ }_{i y s}-1\right] / } \\
& {\left[\mathrm{PO}_{i y s}+\mathrm{Pl}_{\text {iys }}{ }^{*} \mathrm{RR}^{1}{ }_{\text {iys }}+\mathrm{P}^{2}{ }_{i y s}{ }^{*} \mathrm{RR}^{2}{ }_{i y s}\right] }
\end{aligned}
$$

where $\mathrm{P}_{0}$ is prevalence rate of non-smoking; $\mathrm{P}_{1}$ is prevalence rate of current smoking; $\mathrm{P}_{2}$ is prevalence rate of ex-smoking; $\mathrm{RR}_{1}$ is relative mortality rate for current smokers compared to non-smokers; $\mathrm{RR}_{2}$ is relative mortality rate for ex-smokers compared to non-smokers; $y$ is age, categorised into three groups, 35-49, 50-64, and $\geqslant 65$ years; and s is sex.

Prevalence rates of current smoking, ex-smoking, and nonsmoking were obtained from the National Health Interview Survey of Taiwan in 2001.." Prevalence rates of current smoking for males aged 35-49 was 57.4\%, $49.3 \%$ for those aged $50-64$, and $43.7 \%$ for those aged 65 and over. For females, those rates were $4.2 \%, 3.8 \%$, and $2.7 \%$ for the corresponding age groups. Prevalence rates of ex-smoking for males aged $35-49$ was $6.2 \%, 9.0 \%$ for those aged $50-64$, and $20.3 \%$ for those aged 65 and over. For females, those rates were $0.4 \%, 0.6 \%$, and $1.2 \%$, respectively.

The RRs of current smokers compared to non-smokers for each disease by sex and age groups came from the combined cohort mentioned earlier. ${ }^{5-7}$ Only those diseases with RR $>1$ (and $\mathrm{p}<0.05$ ) for current smokers compared to nonsmokers were included in the study. These diseases were then grouped into 19 categories (table 1). In this study, because RRs of ex-smokers compared to non-smokers were not significant, the RRs of ex-smokers were set to 1 and the SAF of ex-smokers became zero.

\section{Estimating the smoking attributable medical expenditures (SAEs)}

The SAEs should include all the medical expenditures incurred due to smoking. However, this study included only outpatient and inpatient expenditures paid by the NHI. This is because the data released by the Bureau of NHI does not include the expenditures not covered by the insurance (for example, certain preventive services, and out-of-plan services).

To estimate the quantity and prices of medical care incurred, we used the International classification of diseases, $9^{\text {th }}$ revision (ICD-9) codes of the 19 categories of diseases as the key variable to link NHI claim data. In terms of estimating morbidity costs, it would be ideal to use relative morbidity rate of smokers compare to non-smokers. However, since we did not have this data, we used relative mortality rate of each disease as the proxy. SAEs were calculated by the following formula:

$$
\begin{aligned}
\text { SAE }_{\text {iys }}= & {\left[\mathrm{P}(\mathrm{MD})_{\text {iys }} \times \mathrm{Q}(\mathrm{MD})_{\text {iys }}+\right.} \\
& \left.\mathrm{P}(\mathrm{H})_{\text {iys }} \times \mathrm{Q}(\mathrm{H})_{\text {iys }}\right] \times \mathrm{SAF}_{\text {iys }}
\end{aligned}
$$

where $\mathrm{P}(\mathrm{MD})$ is the average expenditure per outpatient visit; $\mathrm{Q}(\mathrm{MD})$ is the number of outpatient visits; $\mathrm{P}(\mathrm{H})$ is the average expenditure per admission; $\mathrm{Q}(\mathrm{H})$ is the number of admissions; $\mathrm{i}$ is the type of disease; $\mathrm{y}$ is categorised into three age groups, $35-49,50-64$, and $\geqslant 65$ years; and $s$ is sex.

\section{Estimating mean annual expenditure of current smokers and non-smokers}

Because we did not have smokers or non-smokers' individual aggregated medical expenditures, we made some assumptions when estimating the mean medical expenditures for current smokers and non-smokers. The first assumption was that the basic needs for medical care are the same for current smokers and non-smokers. Thus, the total medical expenditure for current smokers will be the sum of SAE and the basic medical expenditures. The second assumption was that among all the risk factors affecting the use of medical services, smoking condition did not have significant interaction with other risk factors. Therefore, by subtracting SAE from the total medical expenditure, the remaining medical expenditure represents the "basic" expenditures for all the insured people regardless of their smoking condition. We can then determine the amount of this "basic" expenditure for current smokers simply by multiplying the total "basic" expenditure by the prevalence rate of current smoking. Thus mean medical expenditures for each current smoker and non-smoker can be estimated by the following formulae:

Mean medical expenditures of current smokers =

$\left[\right.$ SAEs $+\left(\right.$ BasicExp $\left.\left.\times \mathrm{P}_{1}\right)\right] /\left(\mathrm{N} \times \mathrm{P}_{1}\right)$

where SAEs is smoking attributable expenditures; BasicExp is total medical expenditure of the population SAEs; $\mathrm{P}_{1}$ is prevalence rate of current smoking; and $\mathrm{N}$ is the number of total population.

Mean medical expenditures of non-smokers =

$\left(\right.$ BasicExp $\left.\times \mathrm{P}_{0}\right) /\left(\mathrm{N} \times \mathrm{P}_{0}\right)=$ BasicExp $/ \mathrm{N}$

where BasicExp is the total medical expenditure of the population SAEs; $\mathrm{P}_{0}$ is prevalence rate of non-smoking; and $\mathrm{N}$ is the number of total population.

\section{Estimating smoking attributable deaths, smoking attributable years of potential life lost (YPLL), and smoking attributable cost of premature death}

Smoking attributable deaths were calculated through multiplying estimates of smoking attributable proportion of deaths by total mortality for adults aged 35 and over. However, deaths attributable to fire and exposure to second hand smoke that were accounted in SAMMEC II (smoking attributable mortality, morbidity, and economic costs software, release II) were not included in our study. ${ }^{12}$

Smoking attributable years of potential life lost (YPLL) were estimated according to age and sex specific life expectancy. Future earnings by sex and five year age groups was calculated based on 2001's official statistics related to labour force participation rates, unemployment rates, and the average annual income of those who were employed. Since the growth rates of both the average annual income and gross domestic product (GDP) from 1991 to 2000 happened to be the same at $6 \%$, we used it as the annual increasing rate when estimating future earnings. When discounting future earnings, we used 3\% as the discounting rate as suggested by Max et al. ${ }^{12}$

Smoking attributable deaths (SAD), smoking attributable YPLL (SAYPLL), and smoking- attributable cost of premature deaths (SAPDC) by sex, age group, and disease were estimated according to the following formulae:

$$
\begin{aligned}
& \mathrm{SAD}_{\text {iys }}=\Sigma\left[\mathrm{Q}(\mathrm{D})_{\text {iys }}\right] \times \mathrm{SAF}_{\text {iys }} \\
& \text { SAYPLL }_{\text {iys }}=[\Sigma \mathrm{Q}(\mathrm{D}) \text { igs } \times \mathrm{LEgs}] \times \mathrm{SAF}_{\text {iys }} \\
& \text { SAPDC }_{\text {iys }}=\left[\Sigma \mathrm{Q}(\mathrm{D}) \text { igs } \times \mathrm{LE}_{\mathrm{gs}} \times \mathrm{PVFE}_{\mathrm{gs}}\right] \times \mathrm{SAF}_{\mathrm{iys}}
\end{aligned}
$$

where $\mathrm{i}$ is type of diseases; $\mathrm{y}$ is categorised into three age groups, $35-49,50-64$, and $\geqslant 65$; $g$ is categorised into seven age groups, 35-39, 40-44, 45-49, 50-54, 55-59, 60-64 and $\geqslant 65$; $\mathrm{s}$ is sex; $\mathrm{Q}(\mathrm{D})$ is number of deaths; $\mathrm{LE}_{\mathrm{gs}}$ is life expectancy, if died at age group g; and $\mathrm{PVFE}_{\mathrm{gs}}$ is present value of future earnings, if died at age group g, using a 3\% discount rate and a $6 \%$ annual income growth rate. 


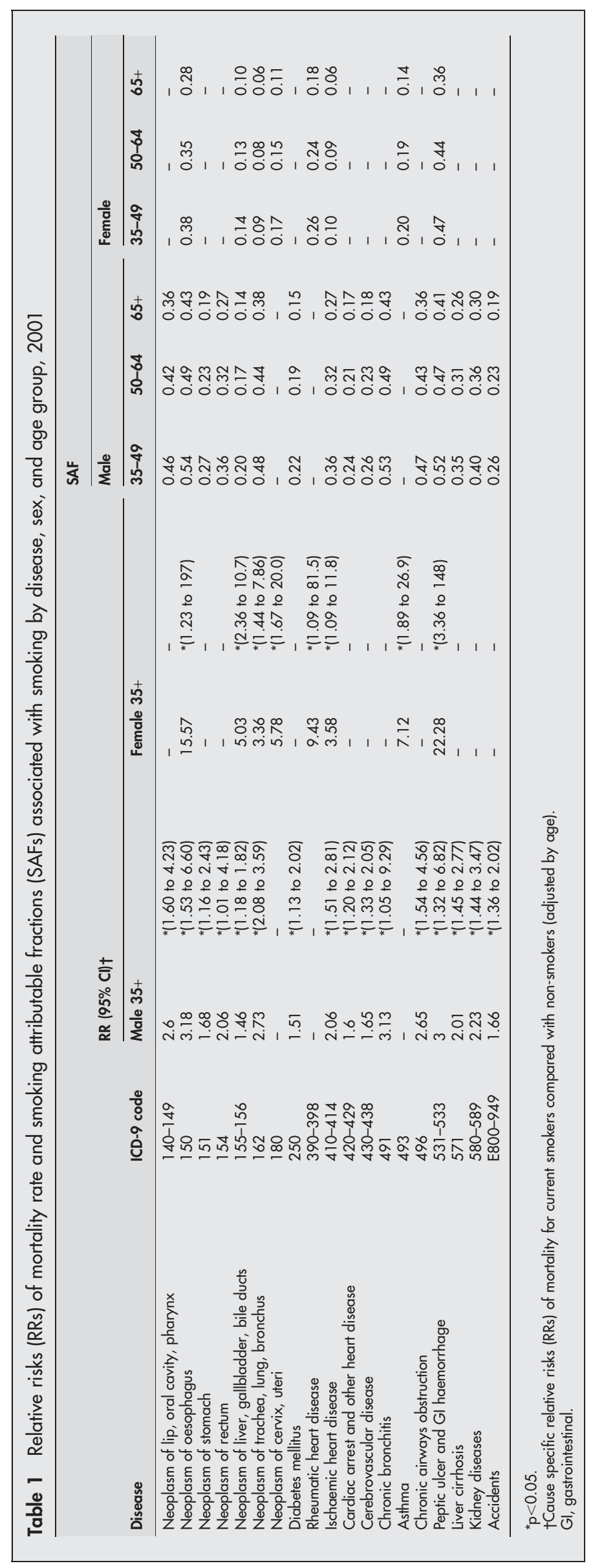




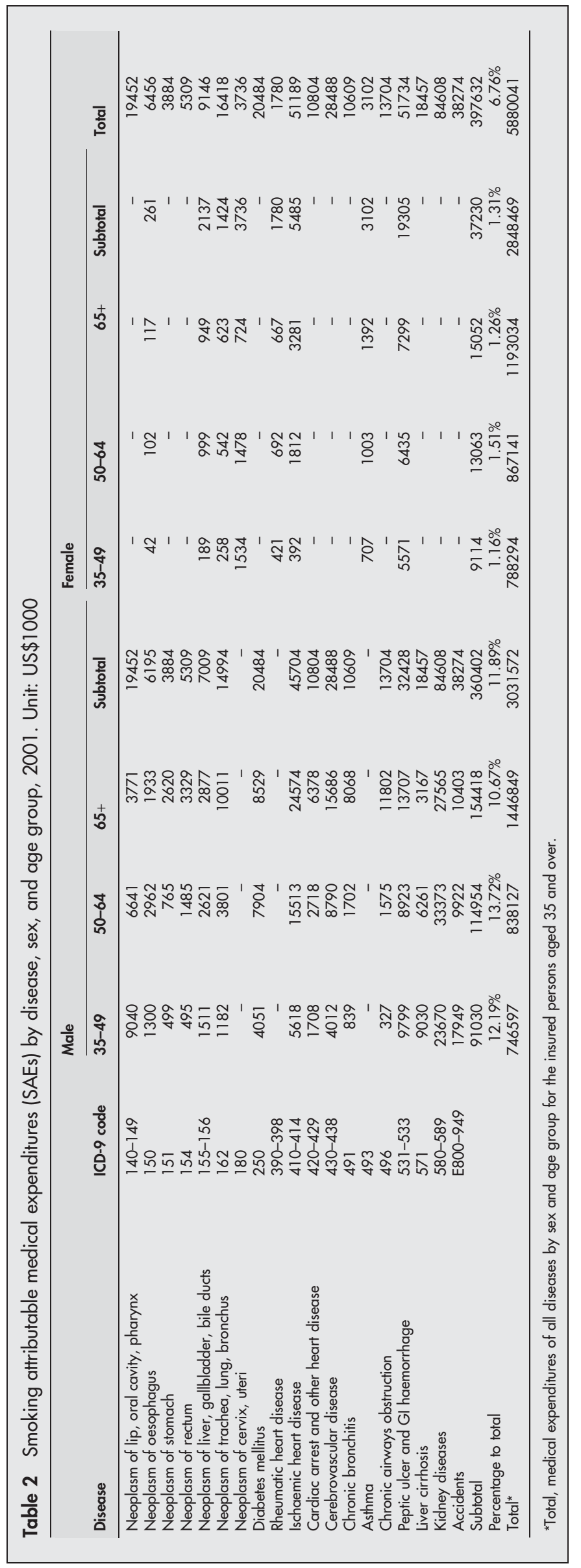


Table 3 Smoking attributable medical expenditures (SAEs) by disease, service type, and age group, 2001. Unit: US\$1000

\begin{tabular}{|c|c|c|c|c|c|c|c|c|c|}
\hline \multirow[b]{2}{*}{ Disease } & \multirow[b]{2}{*}{ ICD-9 code } & \multicolumn{4}{|l|}{ Outpatient } & \multicolumn{4}{|l|}{ Inpatient } \\
\hline & & $35-49$ & $50-64$ & $65+$ & Subtotal & $35-49$ & $50-64$ & $65+$ & Subtotal \\
\hline Neoplasm of lip, oral cavity, pharynx & $140-149$ & 4119 & 2235 & 2155 & 8510 & 4921 & 4406 & 1616 & 10942 \\
\hline Neoplasm of oesophagus & 150 & 122 & 1141 & 233 & 1496 & 1221 & 1923 & 1816 & 4960 \\
\hline Neoplasm of stomach & 151 & 198 & 185 & 686 & 1069 & 301 & 580 & 1933 & 2815 \\
\hline Neoplasm of rectum & 154 & 108 & 599 & 1648 & 2355 & 387 & 887 & 1681 & 2954 \\
\hline Neoplasm of liver, gallbladder, bile ducts & $155-156$ & 289 & 898 & 1073 & 2259 & 1412 & 2723 & 2753 & 6887 \\
\hline Neoplasm of trachea, lung, bronchus & 162 & 483 & 1626 & 3845 & 5953 & 958 & 2717 & 6790 & 10465 \\
\hline Neoplasm of cervix, uteri & 180 & 933 & 926 & 369 & 2229 & 601 & 551 & 355 & 1507 \\
\hline Diabetes mellitus & 250 & 3307 & 6649 & 6858 & 16813 & 744 & 1255 & 1671 & 3670 \\
\hline Rheumatic heart disease & $390-398$ & 116 & 288 & 254 & 657 & 305 & 404 & 413 & 1123 \\
\hline Ischaemic heart disease & $410-414$ & 1926 & 5741 & 9629 & 17296 & 4084 & 11584 & 18225 & 33893 \\
\hline Cardiac arrest and other heart disease & $420-429$ & 702 & 1203 & 2673 & 4578 & 1006 & 1515 & 3705 & 6226 \\
\hline Cerebrovascular disease & $430-438$ & 1679 & 4440 & 7645 & 13764 & 2333 & 4350 & 8042 & 14724 \\
\hline Chronic bronchitis & 491 & 731 & 1204 & 3438 & 5374 & 108 & 497 & 4630 & 5235 \\
\hline Asthma & 493 & 555 & 693 & 620 & 1868 & 152 & 310 & 772 & 1234 \\
\hline Chronic airways obstruction & 496 & 197 & 776 & 3982 & 4955 & 130 & 798 & 7820 & 8748 \\
\hline Peptic ulcer and GI haemorrhage & $531-533$ & 12227 & 11254 & 10758 & 34238 & 3142 & 4105 & 10248 & 17495 \\
\hline Liver cirrhosis & 571 & 5072 & 3597 & 1817 & 10485 & 3958 & 2664 & 1349 & 7972 \\
\hline Kidney diseases & $580-589$ & 22710 & 31922 & 24531 & 79163 & 960 & 1450 & 3034 & 5445 \\
\hline Accidents & E800-949 & 7736 & 4143 & 3319 & 15198 & 10213 & 5779 & 7084 & 23076 \\
\hline Subtotal & & 63209 & 79520 & 85532 & 228261 & 36936 & 48497 & 83937 & 169371 \\
\hline Percentage to total & & $5.96 \%$ & $6.86 \%$ & $5.77 \%$ & $6.16 \%$ & $7.80 \%$ & $8.87 \%$ & $7.26 \%$ & $7.78 \%$ \\
\hline Total $^{*}$ & & 1061280 & 1158349 & 1483350 & 3702979 & 473611 & 546918 & 1156533 & 2177062 \\
\hline
\end{tabular}

*Total, medical expenditure of all diseases for the insured aged 35 and over, by age group and service type.

\section{RESULTS}

Table 1 shows the SAFs of smoking related diseases by sex and age groups. The disease SAFs for males, regardless of age group, were all over $50 \%$ in neoplasm of the oesophagus, chronic bronchitis, and peptic ulcer and gastrointestinal (GI) haemorrhage. On the contrary most SAFs for females were lower than $20 \%$, except for neoplasm of the oesophagus, rheumatic heart disease, asthma, and peptic ulcer and GI haemorrhage. This is because the SAFs were determined by prevalence rate of smoking and RR of each disease. The relatively lower prevalence rate of smoking in females resulted in lower SAFs.

Table 2 shows the SAEs of each disease by sex and age group. Totally, the SAE of smokers was US\$397.6 million, which accounted for $6.8 \%$ of the total medical expenditures of the NHI. The expenditure for kidney disease cost the most, which amounted to US\$84.6 million, followed by peptic ulcer and GI haemorrhage (US\$51.7 million), rheumatic heart disease (US\$51.2 million), accidents (US\$38.3 million), cerebrovascular disease (US\$28.5 million), neoplasm of the lip, oral cavity and pharynx (US\$19.5 million), and liver cirrhosis (US\$18.5 million). When aggregating those seven types of neoplasm into one group, the SAE of neoplasm amounted to US\$64.4 million, accounting for the second largest part of all SAEs.
In terms of the percentages of SAEs in the total amount of medical expenditures paid by the NHI, it was $11.9 \%$ for males and $1.3 \%$ for females. Among males, the percentage of SAEs for persons aged 50-64 was the highest. As for females, persons aged $\geqslant 65$ had the highest percentage.

Table 3 shows the total SAEs of both outpatient and inpatient expenditures by disease. Outpatient expenditures were slightly higher than inpatient expenditures (US\$228.3 million $v \$ 169.4$ million). The percentage of SAEs in the total outpatient expenditure was slightly lower than that of the inpatient expenditure $(6.2 \% \vee 7.8 \%)$. Most of the smoking attributable outpatient expenditures were spent on kidney diseases, especially on haemodialysis. On the other hand, ischaemic heart disease accounted for the highest amount for inpatient services.

Table 4 shows mean annual medical expenditure for current smokers and non-smokers. The mean annual medical expenditures for males were higher for current smokers than that of non-smokers across all age groups. Male current smokers aged 65 years and over had the highest expenditures. For current smokers in this group, outpatient expenditure was US\$908 per person per year and inpatient expenditures was US\$809, yielding a total expenditure of US\$1717 per year. The corresponding amount for nonsmokers was US\$1278. Thus, on the average a current

Table 4 Mean annual medical expenditure of current smokers and non-smokers, 2001. Unit: US\$

\begin{tabular}{|c|c|c|c|c|c|c|c|c|c|}
\hline & \multicolumn{4}{|l|}{ Male } & \multicolumn{4}{|l|}{ Female } & \multirow[b]{2}{*}{ Total } \\
\hline & $35-49$ & $50-64$ & $65+$ & Subtotal & $35-49$ & $50-64$ & $65+$ & Subtotal & \\
\hline \multicolumn{10}{|l|}{ Outpatient } \\
\hline Current smoker & 203 & 454 & 907 & 370.97 & 277 & 605 & 1028 & 469.02 & 378.17 \\
\hline Non-smoker & 163 & 338 & 684 & 324.98 & 215 & 441 & 751 & 380.17 & 252.11 \\
\hline \multicolumn{10}{|l|}{ Inpatient } \\
\hline Current smoker & 110 & 268 & 809 & 253.22 & 100 & 249 & 824 & 238.14 & 363.09 \\
\hline Non-smoker & 85 & 195 & 594 & 225.40 & 80 & 166 & 503 & 184.40 & 197.08 \\
\hline \multicolumn{10}{|l|}{ Total } \\
\hline Current smoker & 312 & 721 & 1717 & 624.19 & 377 & 854 & 1852 & 707.16 & 630.29 \\
\hline Non-smoker & 248 & 533 & 1278 & 550.38 & 295 & 607 & 1254 & 564.57 & 560.18 \\
\hline Difference & 64 & 189 & 439 & 73.81 & 82 & 247 & 598 & 142.59 & 70.11 \\
\hline
\end{tabular}




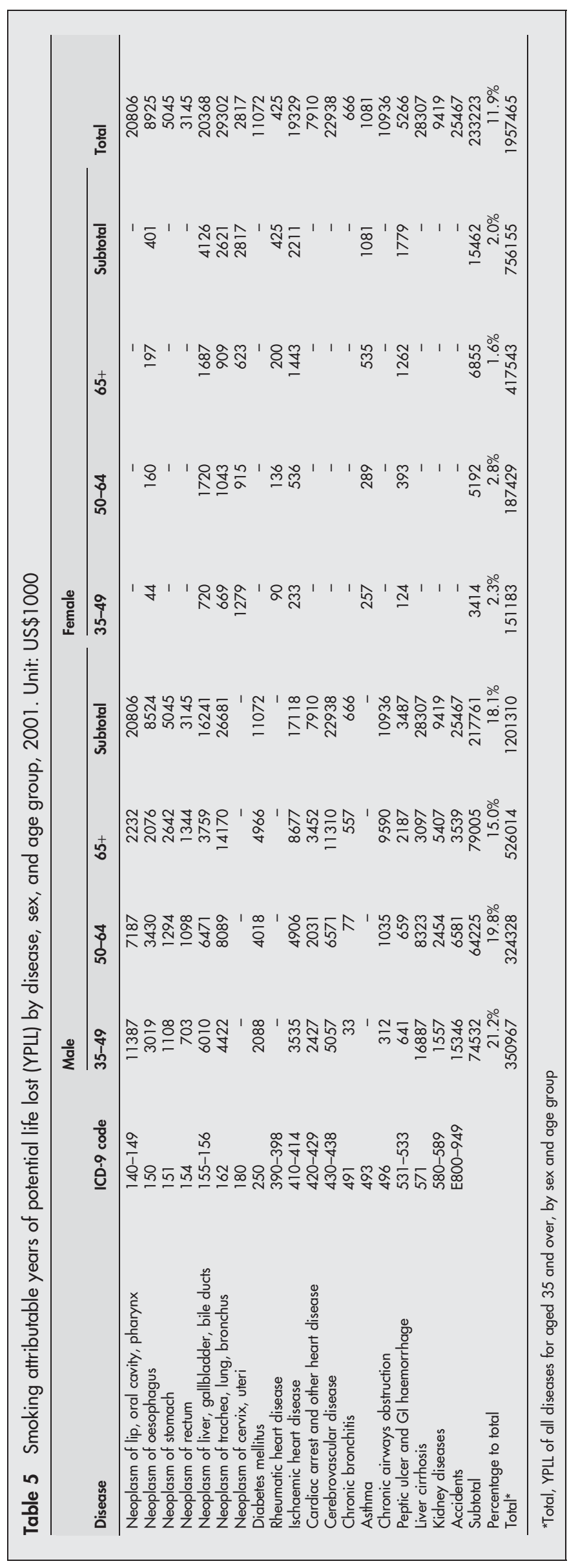




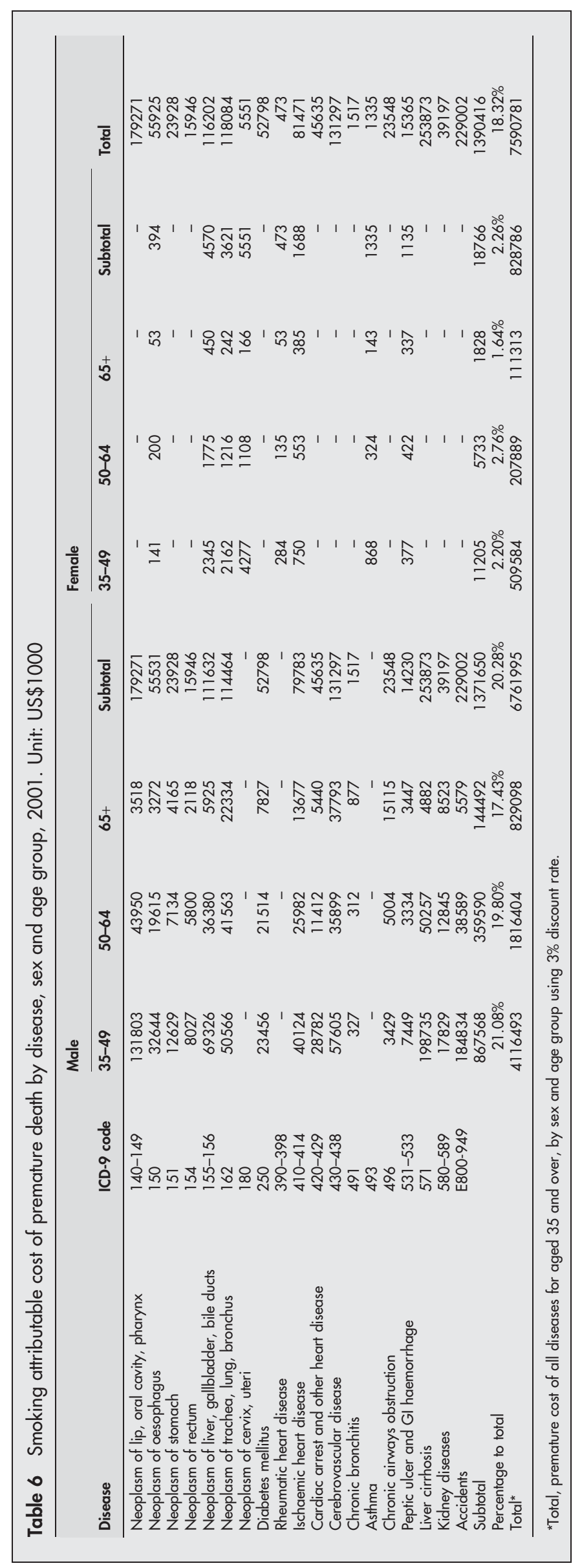

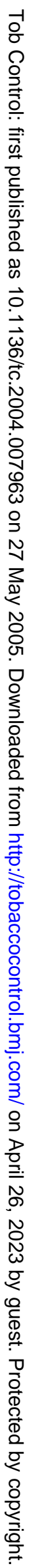


Table 7 Summary table of total smoking attributable cost by sex and age group, 2001. Unit: US\$1000

\begin{tabular}{|c|c|c|c|c|c|c|c|c|c|}
\hline \multirow[b]{2}{*}{ Type of cost } & \multicolumn{4}{|l|}{ Male } & \multicolumn{4}{|l|}{ Female } & \multirow[b]{2}{*}{ Total } \\
\hline & $35-49$ & $50-64$ & $65+$ & Subtotal & $35-49$ & $50-64$ & $65+$ & Subtotal & \\
\hline \multicolumn{10}{|c|}{ Smoking attributable medical expenditures } \\
\hline Outpatient & 56272 & 70856 & 78569 & 205696 & 6937 & 8664 & 6964 & 22564 & 228261 \\
\hline Inpatient & 34758 & 44098 & 75849 & 154705 & 2178 & 4400 & 8088 & 14666 & 169371 \\
\hline Subtotal & 91030 & 114954 & 154418 & 360402 & 9114 & 13063 & 15052 & 37230 & 397632 \\
\hline \multicolumn{10}{|c|}{ Smoking attributable cost of premature death } \\
\hline & 867568 & 359590 & 144492 & 1371650 & 11205 & 5733 & 1828 & 18766 & 1390416 \\
\hline Total & 958598 & 474544 & 298910 & 1732052 & 20320 & 18797 & 16879 & 55996 & 1788048 \\
\hline
\end{tabular}

smoker aged 65 years or over spent US\$439 (\$1717 v \$1278) more than a non-smoker in the same age group. Similarly, females in the same age group had the highest mean annual medical expenditures. A current smoker in this group spent US\$1852 a year while a non-smoker spent US\$1254. Thus, on the average, a female smoker aged 65 or over spent US\$598 more (\$1852 v \$1254) than a non-smoker in the same age group.

Table 5 shows smoking attributable years of potential life lost (SAYPLL). It was 217761 for males and 15462 for females, and accounted for $18 \%$ of males' YPLL and $12 \%$ of females' YPLL due to all causes of death in 2001. All SAYPLL due to seven types of neoplasm amounted to 80441 for males and 9965 for females, and accounted for $37 \%$ and $64 \%$ of total SAYPLL for males and females, respectively. Both for males and females, the SAYPLL due to liver cirrhosis and neoplasm of the trachea, lung, and bronchus were the two highest. Because of the high mortality rate in the oldest age group, it is reasonable to see that this age group had the highest SAYPLL. However, we need to pay more attention to the prevention of deaths from smoking related diseases for males in the younger age group of 35-49 years, because their SAYPLL ranked the second. When examined closely, we found that about $60 \%$ of the SAYPLL in this subgroup was due to three types of diseases, namely liver cirrhosis, accident, and neoplasm of the lip, oral cavity and pharynx.

Table 6 shows the smoking attributable cost of premature death. The total amount of smoking attributable cost of premature death was US\$1371 million for males and US\$18.8 million for females. For both males and females, age group 35-49 ranked the highest, followed by aged 50-64, and aged $\geqslant 65$ ranked the last. Because of higher SAYPLL and higher labour force participation rates, it is not surprising that the younger male group accounted for much of the cost. Although we neglected the income from the underground economy and opportunity cost of housekeeping would underestimate the cost of premature death for females, it is not a major problem in this study because most of the SAFs for females are zero or trivial.

Table 7 summarises the results of this study. The total smoking attributable cost was US\$1.79 billion in 2001 in Taiwan. In terms of type of cost, smoking attributable cost of premature death accounted for a very large proportion. As for sex, males accounted for the most part of the cost due to a much higher rate of smoking. With respect to age groups, younger group had lower medical expenditures, but higher cost of premature death.

\section{DISCUSSION}

Compared to our earlier estimations on SAEs in Taiwan, this study represents a major improvement in two ways. First, the RR of current smokers versus non-smokers was provided by a large combined cohort study that provided comprehensive smoking exposure and mortality data. Second, the amount of medical care utilised by each type of disease and their corresponding expenditures came from the NHI claim files. These claims represented the utilisation of about $97 \%$ of the total population. Therefore the current estimation of SAEs was more precise.

In terms of approaches used to estimate the cost of smoking, Warner $e^{2} a^{13}$ suggests that there are two major types in recent years. One is based on epidemiological findings, using attributable risks as the link between smoking attributable diseases and smoking attributable impacts, such as deaths and medical expenditures. Another approach is to directly collect smokers' and non-smokers' mortality and medical utilisation data. This study adopted the former approach due to limited data and time.

However, both of these approaches have been used in studies in Asia. For example, in Japan, Izumi et al ${ }^{14}$ used a large population based cohort to collect smoking habit and medical utilisation data. They found that smokers consumed excess medical care. Our study had similar findings.

In South Korea, Kang et al ${ }^{15}$ used both approaches in estimating the economic burden of smoking in South Korea. They found that current smokers spent a lot less direct medical costs than non-smokers according to the all causes approach. In our study, however, we found that current smokers had higher medical costs then non-smokers.

This study also adopted the suggestions made by Kang et $a l^{15}$ that the cumulated effects of smoking on mortality may not appear in people under 35 years old. Thus we did not estimate the SAEs of people under 35 .

With respect to the results in our study, kidney diseases accounted for $29 \%$ of outpatient expenditures for all diseases in males. Since $80 \%$ of renal dialyses were done in institution based haemodialysis centres in Taiwan, it will affect the productivity and quality of life of the patients. Thus, kidney disease should be the main concern when discussing the cost of smoking.

In terms of the percentage of SAEs in total medical expenditure, it was $6.8 \%$ in our study, which was similar to the USA. ${ }^{2}$ However, Warner et $\mathrm{l}^{13}$ suggested that $6-8 \%$ would be an underestimation of the true total medical costs of smoking in the USA. In addition, Zhang et al ${ }^{16}$ reported that the SAEs of Medicare beneficiaries accounted for $11.4 \%$ of hospital care and $5.6 \%$ of ambulatory care in Medicare expenditures. In our study, for the corresponding older age group, smoking accounted for $7.3 \%$ of inpatient expenditures and $5.8 \%$ of outpatient expenditures. The percentages for ambulatory care were quite similar, but the percentage for hospital care was $4 \%$ lower in our study than in the USA. The difference might be due to higher unit price of hospital care in the USA.

Most smoking attributable YPLL were related to lung cancer, ischaemic heart disease, and chronic airway obstruction in the USA during 1995-1999. ${ }^{2}$ In our study, the smoking attributable YPLL for lung cancer and ischaemic 


\section{What this paper adds}

Taiwanese smokers incur excess medical expenditure, amounting to $6.8 \%$ for those aged 35 and over. As National Health Insurance is paid for by the general public, non-smokers in Taiwan share that excess medical expenditure. Each smoker spent an extra US\$70 per year on medical care. Each year Taiwan lost an equivalent of US\$1.4 billion in earning power of smokers, amounting to $0.5 \%$ of gross domestic product.

heart disease were also high, but the highest diseases were liver cirrhosis and neoplasm of the trachea, lung, and bronchus. Therefore, we should pay more attention to diseases of the liver when discussing the adverse effects of smoking.

We also noticed that some diseases showed much higher RRs of mortality rate in Lam's $\mathrm{s}^{17}{ }^{18}$ study than those in our study. These diseases included lung cancer, oesophageal cancer, and chronic obstructive pulmonary disease for males. It may be because Lam used the RRs of patients who had smoked at any point in their lives compared with those who never smoked, while we compared current smokers with those who never smoked.

Finally, there were two limitations in this study. First, due to limited data, we were unable to estimate expenditures caused by passive smoking or expenditures not covered by the NHI, making it possible that the SAEs were underestimated.

Second, the RR of mortality was used to estimate the RR of morbidity for each disease. By doing so, we might underestimate some of the expenditures for patients in the more severe stages of disease or in patients with long term nonfatal sickness, such as the common cold. On the other hand, for fatal diseases, we might overestimate current smokers' SAEs. We used a simple formula to illustrate the relationship between RR of mortality and RR of morbidity.

Let D, I, and E denote death, incidence and exposure of a fatal disease.

$\mathrm{RR}$ of mortality $=$

$$
\frac{P(D \mid E)}{P(D \mid \bar{E})}=\frac{P(D \mid I, E)^{*} P(I \mid E)+P(D \mid \bar{I}, E)^{*} P(\bar{I} \mid E)}{P(D \mid I, \bar{E})^{*} P(I \mid \bar{E})+P(D \mid I, \bar{E})^{*} P(I \mid \bar{E})}
$$

When $P(D \mid \bar{I}, E)=0, P(D \mid \bar{I}, \bar{E})=0$, and $P(I \mid E) / P(I \mid \bar{E})=\mathrm{RR}$

of morbidity.

morbidity.
In this case, the value of $\frac{P(D \mid I, E)}{P(D \mid I, \bar{E})}$ will determine whether using RR of mortality as the proxy of RR of morbidity was biased. Because patients with smoking habit are considered to have poor recovery, ${ }^{13}$ the ratio seems to be greater than 1.
However, the absolute value of the ratio will be hard to estimate. In addition, the existence of co-morbidity would complicate this question. Future study may consider to break down the natural history of disease to estimate the difference of transient rate in each state between smokers and non-smokers.

Due to the limitations mentioned above, caution should be taken when using the results of this study.

\section{ACKNOWLEDGEMENTS}

The authors wish to thank the National Health Research Institute for providing National Health Insurance claims data. We would also like to thank Dr Chien-Jen Chen for providing the community cohort data.

\section{Authors' affiliations}

M C Yang, C Y Fann, Institute of Health Care Organization

Administration, National Taiwan University, Taipei, Taiwan

C P Wen, T Y Cheng, Division of Health Policy Research, National Health Research Institutes, Taipei, Taiwan

Competing interests: none declared

\section{REFERENCES}

1 World Health Organization. The World Health Report 1999. Chapter 5. Combating the Tobacco Epidemic 1999:65-79.

2 Centers for Disease Control and Prevention. Annual smoking-attributable mortality, years of potential life lost, and economic costs - United States, 1995-1999. MMWR Morb Mortal Wkly Rep 2002;51:300-3.

3 Cutler DM, Gruber J, Hartman RS, et al. The economic impacts of the tobacco settlement. Journal of Policy Analysis and Management 2002;21:1-19.

4 Warner KE. The economics of tobacco: myths and realities. Tobacco Control 2000;9:78-89

5 Liaw KM, Chen CJ. Mortality attributable to cigarette smoking in Taiwan, a 12-year follow up study. Tobacco Control 1998;7:141-8.

6 Ho MS. A long-term follow-up study of chronic diseases among civil servant and teachers in Taiwan. The Project Report. Taiwan: Institute of Biomedical Sciences, Academia Sinica, 1993

7 Wen CP, Tsai SP, Chen CJ, et al. The mortality risk of smokers in Taiwan. Part I: cause-specific mortality. Prev Med 2004;39:528-35.

8 Rice DP, Hodgson TA, Sinsheimer P, et al. The economic costs of the health effects of smoking, 1984. The Milbank Quarterly 1986;64:489-547.

9 Lilienfeld AM, Lilienfeld DE. Foundations of epidemiology, 3rd ed. New York: Oxford University Press, 1994.

10 Shultz JM, Novotny TE, Rice DP. Quantifying the disease impact of cigarette smoking with SAMMEC II software. Public Health Reports 1991;106:326-33.

11 National Health Interview Survey (NHIS) in 2001. Bureau of Health Promotion and National Health Research Institutes, 2002. hittp://nhis.nhri.org.tw.

12 Max W, Rice DP, Sung HY, et al. Valuing human life: estimating the present value of lifetime earning, 2000. http://repositories.cdlib.org/ctcre/esarm/ PVLE2000.

13 Warner KE, Hodgson TA, Carroll CE. Medical costs of smoking in the United States: estimates, their validity, and their implications. Tobacco Control 1999:8:290-300.

14 Izumi Y, Tsuji I, Ohkubo T, et al. Impact of smoking habit on medical care use and its costs: a prospective observation of National Health Insurance beneficiaries in Japan. Int J Epidemiol 2001;30:616-21.

15 Kang HY, Kim HJ, Park TK, et al. Economic burden of smoking in Korea. Tobacco Control 2003;12:37-44.

16 Zhang X, Miller L, Max W, et al. Cost of smoking to the Medicare Program, 1993. Health Care Financing Review 1999;20:179-96.

17 Lam TH, Ho SY, Hedley AJ, et al. Mortality and smoking in Hong Kong: casecontrol study of all adult deaths in 1998. BMJ 2001;323:361.

18 Lam TH, He YP, Li LS, et al. Mortality attributable to cigarette smoking in China. JAMA 1997;278:1505-8. 